\title{
Welcome to Journal of Food Chemistry and Nanotechnology
}

\author{
Mun Yhung Jung \\ College of Food Science, Woosuk University, Republic of Korea
}

Correspondence to:

Mun Yhung Jung, $\mathrm{PhD}$

College of Food Science

Woosuk University, Samnye-eup

Wanju-gun, Jeonbuk Province 565-701

Republic of Korea

Tel: 82-63-290-1438

Fax: 82-63-291-9312

E-mail: munjung@woosuk.ac.kr

Received: June 09, 2015

Accepted: June 17, 2015

Published: June 19, 2015

Citation: Jung MY. 2015. Welcome to Journal of Food Chemistry and Nanotechnology. J Food Chem Nanotechnol 1(1): 1-2.

Copyright: (C) 2015 Jung. This is an Open Access article distributed under the terms of the Creative Commons Attribution 4.0 International License (CC-BY) (http://creativecommons.org/licenses/ by/4.0/) which permits commercial use, including reproduction, adaptation, and distribution of the article provided the original author and source are credited.

Published by United Scientific Group

\section{Editorial}

With this inaugural issue, it is a great pleasure for me to announce the launch of a new open access journal, Journal of Food Chemistry and Nanotechnology (JFCN). I greatly appreciate the editorial board members who gave us a lot of support, advice, and contribution. Their great expertise and international reputation in the field will surely provide a significant contribution in shaping up JFCN as a world leading platform for the outlet of outstanding research results in the field.

The aim of JFCN is to establish a premier community for publishing high quality papers with significant new research findings and technologies, and technological improvements in the fields of food chemistry and food nanotechnology.

The environments in food research and product development are fast changing with the continuous evolutions of new technologies due to the constant demands for higher quality, and safer and longer shelf-life food products. Food nanotechnology is a good example of the innovation and new technology, which has already begun to be applied in the various foods to meet the high demands. In such a fast changing environment, we need to create a strong community for the effective communication by fast global dissemination of high quality papers in such a new sector. To my knowledge, this is the first journal covering food chemistry along with food nanotechnology, a newly evolving sector in the food science field.

JFCN covers the broad range subjects in food chemistry and food nanotechnology. The subjects include chemical and enzymatic interaction of the components of various foods, food structure and functionality, analytical method developments, food phytochemicals, flavors, additives, colorants, nutrients, toxicants, food packaging, processing, fermentation, probiotics, nanocapsules, nanotubes and nanoparticles, nanoemulsions, nanosensors, and nanoclays and nanoflims as barrier materials for food packaging.

JFCN will publish only rigorously peer-reviewed, high quality papers with strong impact across the entire spectrum of the field. JFCN will ensure full use of open-access journal in the rapidly changing environment of publication and dissemination of scientific articles.

We will strive to make JFCN a world class scientific journal for the rapid publication, effective communication, and significant scientific advances in the food science sector.

I encourage you to join us as active authors, voluntary reviewers and editorial board members in this creative process. The submitted manuscripts will be strictly 
reviewed in a timely and unbiased manner to ensure the rapid publication as well as the quality of the papers. Submission of your full-length research articles, short communication, mini- or comprehensive review papers, letters, and critical commentaries to JFCN will be greatly appreciated. Any suggestion and opinion regarding the journal activity will be always welcome. I hope that JFCN will be the main outlet of high impact research works carrying significant new findings, improvement, innovations, and new technology in the field.

Mun Yhung Jung

Professor

Woosuk University 